\title{
POSITIVE $p$-SUMMING OPERATORS, VECTOR MEASURES AND TENSOR PRODUCTS
}

\author{
by OSCAR BLASCO \\ (Received 25th February 1986)
}

\section{Introduction}

In this paper we shall introduce a certain class of operators from a Banach lattice $X$ into a Banach space $B$ (see Definition 1) which is closely related to $p$-absolutely summing operators defined by Pietsch [8].

These operators, called positive p-summing, have already been considered in [9] in the case $p=1$ (there they are called cone absolutely summing, c.a.s.) and in [1] by the author who found this space to be the space of boundary values of harmonic $B$-valued functions in $h_{B}^{p}(D)$.

Here we shall use these spaces and the space of majorizing operators to characterize the space of bounded $p$-variation measures $V_{B}^{p}$ and to endow the tensor product $L^{p} \otimes B$ with a norm in order to get $L^{p}(B)$ as its completion in this norm.

\section{Some definitions and previous results}

Throughout this paper $X$ will denote a Banach lattice and $B$ a Banach space. Given $1 \leqq p \leqq \infty$ we shall always write $p^{\prime}$ for such a number that $(1 / p)+\left(1 / p^{\prime}\right)=1$.

Definition 1. An operator $T$ belonging to $L(X, B)$ is called positive p-summing $(1 \leqq p<\infty)$ if there exists a constant $C>0$ such that for all positive elements $x_{1}, x_{2}, \ldots, x_{n}$ in $X$ we have

$$
\left(\sum_{i=1}^{n}\left\|T x_{i}\right\|_{B}^{p}\right)^{1 / p} \leqq C \cdot \sup _{\|\xi\|_{x^{*}} \leqq 1}\left(\sum_{i=1}^{n}\left|\left\langle\xi, x_{i}\right\rangle\right|^{p}\right)^{1 / p}
$$

We shall denote by $\Lambda_{p}(X, B)$ the space of such operators and the infimum of the constants will be the norm on it.

A duality argument allows us to write the following equivalent formulation of (1):

$$
\left(\sum_{i=1}^{n}\left\|T x_{i}\right\|_{B}^{p}\right)^{1 / p} \leqq C \cdot \sup \left\{\left\|\sum_{i=1}^{n} \alpha_{i} \cdot x_{i}\right\|_{X}: \sum_{i=1}^{n} \alpha_{i}^{p^{\prime}} \leqq 1, \alpha_{i} \geqq 0\right\} .
$$

Obviously the space of $p$-absolutely summing operators $\Pi_{p}(X, B)$ is included in $\Lambda_{p}(X, B)$ and the same techniques as for $p$-absolutely summing operators lead us to see 
that for $p \leqq q, \Lambda_{p}(X, B) \subseteq \Lambda_{q}(X, B)$ and

$$
|T|_{\Lambda_{q}} \leqq|T|_{\Lambda_{p}} \text { for all } T \text { in } \Lambda_{p}(X, B) .
$$

Definition 2 (see [9]). An operator $T$ belonging to $L(B, X)$ is called majorizing if there exists a constant $C>0$ such that for every $x_{1}, x_{2}, \ldots, x_{n}$ in $B$

$$
\left\|\sup _{1 \leqq i \leqq n}\left|T x_{i}\right|\right\|_{X} \leqq C \cdot \sup _{1 \leqq i \leqq n}\left\|x_{i}\right\|_{B}
$$

We shall denote by $M(B, X)$ the space of such operators and we shall set the following norm on it:

$$
|T|_{m}=\sup \left\{\left\|\sup _{1 \leqq i \leqq n}\left|T x_{i}\right|\right\|_{X}:\left\{x_{i}\right\} \in B,\left\|x_{i}\right\|_{B} \leqq 1\right\} .
$$

If we consider $A \otimes B$ as a subspace of $U\left(A^{*}, B\right)$, that is $u=\sum_{i=1}^{n} a_{i} \otimes b_{i}$ represents the operator $T_{u}$ defined by $T_{u}(\xi)=\sum_{i=1}^{n}\left\langle\xi, a_{i}\right\rangle \cdot b_{i}$, then it is easy to see that $A \otimes B$ is included in $\Lambda_{p}\left(A^{*}, B\right)$ and $M\left(A^{*}, B\right)$. Let us denote by $A \hat{\otimes}_{p} B$ and $A \check{\otimes}_{m} B$ the completion of the space $A \otimes B$ endowed with the norms induced by $\Lambda_{p}\left(A^{*}, B\right)$ and $M\left(A^{*}, B\right)$ respectively.

\section{Applications to tensor products and vector measures}

Let $(\Omega, \mathscr{B}, \mu)$ be a finite measure space and $1 \leqq p<\infty$. We shall denote by $L^{p}(\mu, B)$ the space of measurable functions such that $\|f\|_{p}=\left(\int_{\Omega}\|f(t)\|^{p} d \mu\right)^{1 / p}<+\infty$.

The following result can be found in [9].

$$
L^{p}(\mu) \hat{\otimes}_{1} B=L^{p}(\mu, B) \quad 1 \leqq p<\infty .
$$

This fact can be extended in the following way:

Theorem 1. Let $1 \leqq p<\infty$, then for all $1 \leqq r \leqq p$

$$
L^{p}(\mu) \hat{\otimes}, B=L^{p}(\mu, B) .
$$

Proof. Let $1 \leqq r \leqq p$. Since simple functions are dense in $L^{P}(\mu, B)$, it suffices to show that for each $s=\sum_{i=1}^{n} x_{i} \cdot \chi_{E_{i}}$ we have that the operator $T_{s}(\psi)=\int_{\Omega} s(t) \cdot \psi(t) d \mu(t)$ satisfies $\left|T_{s}\right|_{\Lambda}=\|s\|_{p}$.

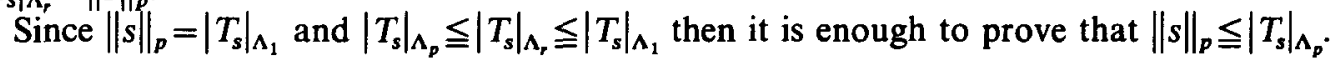

$$
\|s\|_{p}=\left(\sum_{i=1}^{n}\left\|x_{i}\right\|^{p} \cdot \mu\left(E_{i}\right)\right)^{1 / p}
$$




$$
\begin{aligned}
& =\left(\sum_{i=1}^{n}\left\|T_{s}\left(\mu\left(E_{i}\right)^{-1} \cdot \chi_{E_{i}}\right)\right\|^{p} \cdot \mu\left(E_{i}\right)\right)^{1 / p} \\
& =\left(\sum_{i=1}^{n}\left\|T_{s}\left(\mu\left(E_{i}\right)^{-1 / p^{\prime}} \cdot \chi_{E_{i}}\right)\right\|^{p}\right)^{1 / p} \\
& \leqq\left|T_{s}\right|_{\Lambda_{p}} \cdot \sup \left\{\left\|\sum_{i=1}^{n} \alpha_{i} \cdot \mu\left(E_{i}\right)^{-1 / p^{\prime}} \cdot \chi_{E_{i}}\right\|_{L p^{\prime}} \sum_{i=1}^{n} \alpha_{i}^{p^{\prime}} \leqq 1, \alpha_{i} \geqq 0\right\} \\
& =\left|T_{s}\right|_{\Lambda_{p}} .
\end{aligned}
$$

We can give another representation of $\Lambda_{p}\left(L^{p^{\prime}}(\mu), B\right)$ in terms of vector measures.

Let us recall a space of $B$-valued measures, introduced by Bochner [2] in the scalarvalued case, which is a good substitute for $L^{P}(\mu, B)$ in several cases, for example for the duality $\left(L^{D}(\mu, B)\right)^{*}=V_{B^{*}}^{p^{\prime}}$ or for boundary values of functions in $h_{B}^{p}(D)[1]$.

Definition 3. A finitely additive vector measure $G: \mathscr{B} \rightarrow B$ is said to have bounded $p$ variation if

$$
|G|_{p}=\sup _{\pi}\left\{\left(\sum_{E \in \pi} \frac{\|G(E)\|^{p}}{\mu(E)^{p-1}}\right)^{1 / p}\right\}<+\infty \quad(1<p<\infty)
$$

where the "sup" is taken over all finite partitions of $\Omega$ and

$$
|G|_{\infty}=\sup \left\{\frac{\|G(E)\|}{\mu(E)}, E \in \mathscr{B}\right\}<+\infty \quad(p=\infty) .
$$

We shall denote by $V_{B}^{p}$ the space of such measures and its norm is given by (5) or ( $\left.5^{\prime}\right)$ provided $1<p<\infty$ or $p=\infty$.

Let us recall some properties of this space.

(a) Every measure in $V_{B}^{p}$ is countably additive, $\mu$-continuous and with bounded variation.

(b) $L^{p}(\mu, B)$ is isometrically embedded in $V_{B}^{p}$.

Dinculeanu [4] characterized the space $V_{B}^{p}$ in terms of $\mathscr{L}\left(L^{p^{\prime}}(\mu), B\right)$, the space of operators in $L\left(L^{\prime}(\mu), B\right)$ such that

$$
\|T\|_{p}=\sup \left\{\sum_{i=1}^{n}\left|\alpha_{i}\right| \cdot\left\|T\left(\chi_{E_{i}}\right)\right\|_{B}:\left\|\sum_{i=1}^{n} \alpha_{i} \cdot \chi_{E_{i}}\right\|_{L^{p}} \leqq 1\right\}<+\infty .
$$

The author proved in [1] that $\mathscr{L}\left(L^{\prime}(\mu), B\right)=\Lambda_{p}\left(L^{\prime}(\mu), B\right)$, hence we have the following:

Theorem 2. For $1<p \leqq \infty, \Lambda_{p}\left(L^{p^{\prime}}(\mu), B\right)=V_{B}^{p}$.

Now we shall characterize $V_{B}^{p}$ by means of the space of certain majorizing operators. 
Theorem 3. For $1<p<\infty, M\left(B, L^{p}(\mu)\right)=V_{B^{*}}^{p}$

Proof. Let $G$ be a measure of $V_{B^{*}}^{p}$ and take $x \in B$ with $\|x\|_{B}=1$. Consider now the measure $G_{x}(E)=\langle G(E), x\rangle$ for all measurable set $E$ and the positive measure $|G|$. Both measures are countably additive, $\mu$-continuous and with bounded variation. So, by the Radon-Nikodým theorem, there exist $f_{x}$ and $g \geqq 0$ in $L^{1}(\mu)$ such that

$$
\begin{array}{ll}
G_{x}(E)=\int_{E} f_{x}(t) d \mu(t) & \text { for all } E \in \mathscr{B}, \\
|G|(E)=\int_{E} g(t) d \mu(t) & \text { for all } E \in \mathscr{B} .
\end{array}
$$

It is not difficult to show, since $G$ belongs to $V_{B}^{p}$, that $f_{x}$ and $g$ belong to $L^{p}(\mu)$ and moreover $\|g\|_{p}=|G|_{p}$ (see the argument in [1, Proposition 3]).

Due to (6) and (7) we have that

$$
\left|G_{x}\right|(E)=\int_{E}\left|f_{x}(t)\right| d \mu(t) \leqq|G|(E)=\int_{E} g(t) d \mu(t)
$$

and from this we obtain

$$
\left|f_{x}(t)\right| \leqq|g(t)| \quad \mu \text {-a.e. }
$$

Let us define $T: B \rightarrow L^{p}(\mu)$

$$
y \mapsto T(y)=\|y\|_{B} \cdot f_{y /\|y\|_{B}}
$$

From (8) it is easy to show that $T \in M\left(B, L^{p}(\mu)\right)$.

Indeed, if $x_{1}, x_{2}, \ldots, x_{n}$ belong to $B$ and $\left\|x_{i}\right\|_{B}=1$ then

$$
\left\|\sup _{1 \leqq i \leqq n}\left|T x_{i}\right|\right\|_{L^{p}} \leqq\|g\|_{p}=|G|_{p^{*}}
$$

Conversely, given $T$ in $M\left(B, L^{p}(\mu)\right)$ and denoting by $f_{x}$ the function $T x$, we can define the measure $G: \mathscr{B} \rightarrow B^{*}$ by

$$
\langle G(E), x\rangle=\int_{E} f_{x}(t) d \mu(t)
$$

Now, let $\pi$ be a partition of $\Omega$. Given $\varepsilon>0$, for each $E \in \pi$ there exists $b_{E} \in B$ with $\left\|b_{E}\right\|_{B}=1$ such that

$$
\mu(E)^{-1 / p^{\prime}} \cdot\|G(E)\| \leqq\left\langle\mu(E)^{-1 / p^{\prime}} \cdot G(E), b_{E}\right\rangle+\varepsilon / n^{1 / p}
$$

From (10) the triangle inequality in $\ell^{p}$ implies

$$
\left(\sum_{E \in \pi}\left(\mu(E)^{-1 / p^{\prime}} \cdot\|G(E)\|\right)^{p}\right)^{1 / p}=\left(\sum_{E \in \pi}\left|\left\langle\mu(E)^{-1 / p^{\prime}} \cdot G(E), b_{E}\right\rangle\right|^{p}\right)^{1 / p}+\varepsilon
$$


Now by using (9) we can write

$$
\begin{aligned}
\left(\sum_{E \in \pi} \frac{\|G(E)\|^{p}}{\mu(E)^{p-1}}\right)^{1 / p} & \leqq\left(\sum_{E \in \pi}\left(\mu(E)^{-1 / p^{\prime}} \cdot\left|\int_{E} f_{b_{E}}(t) d \mu(t)\right|\right)^{p}\right)^{1 / p}+\varepsilon \\
& =\sup _{\Sigma \alpha_{E}^{p^{\prime}}=1}\left\{\sum_{E \in \pi} \int_{E}\left|f_{b_{E}}(t)\right| \cdot \alpha_{E} \cdot \mu(E)^{-1 / p^{\prime}} \cdot d \mu(t)\right\}+\varepsilon \\
& \leqq \sup _{\Sigma \alpha_{E}^{p^{\prime}}=1}\left\{\int_{\Omega}\left(\sup _{E \in \pi}\left|f_{b_{E}}(t)\right|\right)\left(\sum_{E \in \pi} \alpha_{E} \cdot \mu(E)^{-1 / p^{\prime}} \cdot \chi_{E}(t)\right) d \mu(t)\right\}+\varepsilon \\
& \leqq\left\|\sup _{E \in \pi}\left|T\left(b_{E}\right)\right|\right\|_{L p} \cdot \sup _{\Sigma \alpha_{E}^{p^{\prime}=1}}\left\{\left\|\sum_{E \in \pi} \alpha_{E} \cdot \mu(E)^{-1 / p^{\prime}} \cdot \chi_{E}\right\|_{L^{p^{p}}}\right\}+\varepsilon \\
& \leqq|T|_{m}+\varepsilon .
\end{aligned}
$$

Taking $\varepsilon$ arbitrarily small and the "sup" over the partitions we obtain $|G|_{p} \leqq|T|_{m}$, completing the proof.

This theorem allows us to prove the following result of [5].

Corollary. $B \check{\otimes}_{m} L^{p}(\mu)=L^{p}(\mu, B)$ for each $1<p<\infty$.

Proof. Given a simple function $s=\sum_{i=1}^{n} x_{i} \cdot \chi_{E_{i}}$ where $x_{i}$ belongs to $B$, we notice that $s$ clearly belongs to $L^{p}\left(\mu, B^{* *}\right)$ and therefore the measure $G_{s}(E)=\int_{E} s(t) d \mu(t)$ belongs to $V_{B^{* *}}^{p}=M\left(B^{*}, L^{p}(\mu)\right)$. So, denoting by $T_{s}$ the operator associated with $s$ we have $\|s\|_{p}=$ $\left|G_{s}\right|_{p}=\left|T_{s}\right|_{m}$. Finally the density of simple functions in the space $L^{D}(\mu, B)$ gives us the corollary.

\section{REFERENCES}

1. O. BLAsco, Boundary values of vector valued harmonic functions considered as operators, Studia Math. 86 (1987), 19-33.

2. S. Bochner, Additive set functions on groups, Ann. of Math. 40 (1939), 769-799.

3. J. Diestel and J. J. UhL, Vector Measures (Amer. Math. Soc. Mathematical Surveys 15, (1977)).

4. N. Dinculeanu, Vector Measures (Pergamon Press, New York, 1967).

5. S. Heinrich, M. J. Nielsen and G. Olsen, Order bounded operators and tensor products of Banach lattices, Math. Scand. 49 (1981), 99-127.

6. S. Leader, The theory of $L^{p}$-spaces for finitely additives set functions, Ann. of Math. (2) 58 (1953), 528-543.

7. J. Lindenstrauss and L. Tzafiri, Classical Banach Spaces, Vols. I and II (Springer-Verlag, Berlin, 1979). 
8. A. Pietsch, Absolut p-summierende Abbildungen in normmierten Rieumen, Studia Math. 28 (1967), 333-353.

9. H. H. SchaEfFer, Banach Lattices and Positive Operators (Springer-Verlag, Berlin, 1974).

Dpto. Teoria de Funciones

Facultad de Ciencias

50009-ZARAGOZA

SPAIN 